American Journal of Infectious Diseases 3 (1): 7-10, 2007

ISSN 1553-6203

(C) 2007 Science Publications

\title{
Acute and Subacute Phase of Cerebral Infarction After Treatment With Acupuncture and Medical Therapy
}

\author{
${ }^{1}$ Mutasem Abu Hamed, ${ }^{1} \mathrm{Zi}$ Xiao Hong, ${ }^{2}$ Xiao bo, ${ }^{1}$ Song Zhi and ${ }^{1}$ Tu qiu Yun \\ ${ }^{1}$ Department of Neurology, Xiangya third affiliated hospital in Xiangya medical college of Central south \\ university, Changsha, 430013 Hunan, province, P.R China \\ ${ }^{2}$ Department of Neurology, Xiangya first affiliated hospital in Xiangya medical college of Central south \\ university, Changsha, Hunan province, P.R China
}

\begin{abstract}
Stroke is the third leading cause of death in developed countries and the second cause of death in patients aged 65 years or more. Stroke is characterized by the sudden loss of circulation to an area of the brain, resulting in a corresponding loss of neurologic function. The aim of this study is to investigate whether Acupuncture treatment has an additional effect to medical treatment and physiotherapy (passive and active movement) in cerebral infarction and Acupuncture treatment in acute phase cerebral infarction (started 1-3 days post stroke onset) is more effective than Acupuncture in subacute phase cerebral infarction (started 8 days post stroke onset). In this study 126 patients suffering from cerebral infarction were divided into three groups. Two of them are Acupuncture groups (acute cerebral infarction group and subacute cerebral infarction group): receiving Acupuncture in addition to medical treatment and physiotherapy and the third is a (control group): receiving medical treatment and physiotherapy. In each group the patients were divided into Right Handed patients complaining of Right Hemiparalysis (RHRH) and Right Handed patients complaining of Left Hemiparalysis (RHLH). Results showed that motor function and disability were improved in the Acupuncture groups in RHRH $(\mathrm{P}<0.01)$ and were not improved in RHLH $(\mathrm{P}>0.05)$. The improvement in the acute cerebral infarction group was much better than in the subacute cerebral infarction group $(\mathrm{P}<0.01)$.
\end{abstract}

Key Words: cerebral infarction, Acupuncture treatment, Barthel index, NIHSS.

\section{INTRODUCTION}

Acupuncture is one of the main modalities of treatment in traditional Chinese medicine (TCM) and can be traced back more than 2000 years in China. It was widely used to treat hemiplegia long before the Tang dynasty. There are numerous reports in the Chinese literature about the efficacy of acupuncture in stroke rehabilitation. However, the result was rarely quantitatively expressed by properly validated measures.

In recent Studies showing that Acupuncture opens blood vessels for a better flow and decreases clotting and inflammation. These effects are desired early when arteries are blocked ${ }^{[1]}$ Acupuncture may increase blood circulation and body temperature. It may also affect white blood cell activity (responsible for our immune function), reduce cholesterol and triglyceride levels, and regulate blood sugar levels and improve the viscosity of the blood. The biochemical and immunologic properties of blood were affected by Acupuncture ${ }^{[23]}$ In acute stroke - Acute phase (1-7 days post stroke onset) the oedema increases, the ischemic area appears as an area of hypointensity onT1-weighted imaging (T1-WI) and as a hyperintense area on T2weighted imaging (T2-WI). The arterial enhancement usually persists, petechial and frank haemorrhage can be observed, typically 24-48 hours after the onset of the stroke. In Subacute phase (8-21 days post stroke onset) the oedema resolves and the mass effect becomes less appreciated; however, the infracted areas still appear as a hypointensity on T1-WI and as a hyperintensity on $\mathrm{T} 2-\mathrm{W}$ the arterial enhancement is usually resolved by this time, and the parenchymal enhancement typically persists throughout this phase ${ }^{[4]}$

Motor and functional recovery after a stroke varies to a great extent between individuals. A review of the research literature on stroke outcomes indicates that

Corresponding Author: $\quad$ Mutasem Abu Hamed: Department of Neurology, Xiangya third affiliated hospital in Xiang ya medical college of Central south university, Changsha, Hunan province, P.R China 
multiple factors are related to the prognosis of recovery; including age, brain lesion size and lesion location. Accordingly, individuals with dominant-side hemi paresis are typically more motivated to use the dominant limb. In contrast, stroke survivors with nondominant hemiparalysis continue to compensate with the intact, dominant limb ${ }^{[5]}$ Passive movements in hemiplegic stroke patients before clinical recovery elicit some of the brain activation patterns. Changes of cerebral activation in sensory and motor systems occur early after stroke and may be a first step toward restoration of motor function after stroke ${ }^{[6]}$. Constraintinduced therapy improves functional use and expands cortical representation of the exercised limb ${ }^{\text {[7] }}$

The goal of this study is to maximize or restore motor function and functional disability. After local damage to the motor cortex, rehabilitative training can shape subsequent reorganization in the adjacent intact cortex, and that the undamaged motor cortex may play an important role in motor recovery.

\section{MATERIALS AND METHODS}

Study Design: 26 Patients suffering from acute cerebral infarction collected from Xiangya first affiliated Hospital and Xiangya third affiliated hospital in Xiangya medical college of Central south university, Changsha city, Hunan province, P.R China were included in this study from September 2005 to June 2006.

68 Patients were males and 58 were females, Age ranged from $40-80$ years old with a mean 60 years old, $40 \%$ of patient were smokers, $60 \%$ were retired, $5 \%$ were alcohol dependent, and $70 \%$ of patients admitted with blood pressure from $170 / 110 \mathrm{mmHg}$ to $150 / 90 \mathrm{mmHg}$ with a mean $160 / 100 \mathrm{mmHg}$. There were no difference between acute cerebral infarction group, subacute cerebral infarction group and cerebral infarction group (control group) in age, brain lesion size and lesion location $(\mathrm{P}>0.05)$.

Inclusion criteria included acute stroke resulting in hemiparalysis or hemiplegia, diagnosed by neurological examination, CT or MRI scan, confirmed by diagnostic Criteria for acute ischemic cerebrovascular syndrome (AICS) ${ }^{[8]}$ and should limit enrolment to patients with "definite" AICS whenever feasible. Exclusion criteria included (a) history of previous cerebral infarction confirmed with CT or MRI (b) coma (c) severe systemic diseases or life-threaten illness (d) Patients who were not independent in activities of daily living prior to cerebral infarction onset.

Cerebral infarction patients were divided into three groups as shown in table1:

Regular examination of motor function and other routine neurological examination were performed at the admission, three days, one week, two weeks and three weeks post stroke onset.

Outcome measures methods: Outcome measures included Barthel index and NIH scale;

The Barthel Index (BI) is considered to be the best of the activity of daily living (ADL) measurement scales and one of the most reliable instruments of clinical measurement in stroke. It is well known that it shares the fundamental weakness of most ADL scales, that is, their ceiling and floor effect. It contains 10 items with varying weights that score ADL. The items bathing and grooming are scored 0 or 5; the items feeding, dressing, controlling bladder, controlling bowel, getting onto and off the toilet, and ascending and descending stairs are scored 0,5 , or 10 . Items regarding moving from wheelchair to bed and walking on level surface are scored $0,5,10$, or 15 . The total $\mathrm{BI}$ is a cumulative score of the 10 items, with a maximum score of 100 corresponding with complete independence, and a minimum score of 0 corresponding with total dependence ${ }^{[9]}$ A score of 60 appears to be a pivotal score where patients move from dependency to assisted independence ${ }^{[10]}$ Most patients were independent for essential personal care, such as moving around unassisted, sphincter control, eating, and personal toilet. A score of 85 usually corresponded to independence with minimal assistance. This means that the majority of patients were able to get dressed and to move from armchair to bed unassisted.

The National Institutes of Health (NIH) Stroke Scale (NIHSS) is a well validated and commonly used stroke impairment scale that sums the scores from individual elements of the neurological examination to provide an overall stroke impairment score. The NIH Stroke Scale (NIHSS) is accepted widely for measuring acute stroke deficits in clinical trials and has become a standard part of the clinical assessments used in many recent interventional trials ${ }^{[11]}$ It includes items to assess level of consciousness, gaze, visual fields, facial palsy, motor strength, ataxia, sensation, language, dysarthria, and extinction/inattention. It is an attractive candidate predictor of post hospital disposition because it is widely used, is easy to learn, and can be performed rapidly on admission. 
Am. J. Infect. Dis., 3 (1): 7-10, 2007

Table 1: Patient distribution according to treatment and side of hemiparalysis

\begin{tabular}{|c|c|c|c|}
\hline $\begin{array}{l}42 \text { patients with Acute Cerebral } \\
\text { Infarction }(\mathrm{ACI})\end{array}$ & $\begin{array}{l}42 \text { patients with Subacute Cerebral Infarction } \\
\text { (SACI) }\end{array}$ & \multicolumn{2}{|c|}{$\begin{array}{l}42 \text { patients with acute cerebral infarction } \\
\text { (control group) }\end{array}$} \\
\hline $\begin{array}{l}\text { Received Acupuncture } 1 \text { to } 3 \text { days } \\
\text { post acute cerebral infarction, } \\
\text { medical treatment and } \\
\text { physiotherapy }\end{array}$ & $\begin{array}{l}\text { Received Acupuncture } 8 \text { days post acute } \\
\text { cerebral infarction, medical treatment and } \\
\text { physiotherapy }\end{array}$ & & $\begin{array}{l}\text { medical treatment and } \\
\text { iotherapy. }\end{array}$ \\
\hline 27 (RHRH) & 15 (RHLH) & $\begin{array}{l}15 \\
\text { (RHL } \\
\text { H) }\end{array}$ & 27 (RHRH) \\
\hline
\end{tabular}

Table 2: Comparison of motor function

\begin{tabular}{|c|c|c|c|c|c|c|}
\hline \multirow{2}{*}{ Variables } & \multicolumn{2}{|c|}{$\mathrm{ACI}$} & \multicolumn{2}{|c|}{ SACI } & \multicolumn{2}{|c|}{ Control Group } \\
\hline & 27 RHRH & 15 RHLH & 27 RHRH & 15 RHLH & 27 RHRH & 15 RHLH \\
\hline $1^{\text {st }}$ Week & $4.22 \pm 0.75^{*}$ & $4.73 \pm 1.03$ & $5.63 \pm 0.74$ & $5.33 \pm 0.82$ & $5.81 \pm 1.00$ & $5.2 \pm 1015$ \\
\hline $2^{\text {nd }}$ Week & $3.15 \pm 0.53 *$ & $3.53 \pm 1.19$ & $4.29 \pm 0.82 *$ & $4.13 \pm 0.92$ & $5.15 \pm 0.95$ & $4.27 \pm 1.44$ \\
\hline $3^{\text {rd }}$ Week & $2.50 \pm 0.51^{*}$ & $2.80 \pm 0.68$ & $3.00 \pm 0.73 *$ & $2.60 \pm 1.24$ & $4.44 \pm 0.93$ & $3.60 \pm 1.59$ \\
\hline \multirow{2}{*}{ Variables } & \multicolumn{2}{|c|}{ ACI } & \multicolumn{2}{|c|}{ SACI } & \multicolumn{2}{|c|}{ Control Group } \\
\hline & 27 RHRH & 15 RHLH & $27 \mathrm{RHRH}$ & 15 RHLH & 27 RHRH & $15 \mathrm{RHLH}$ \\
\hline $1^{\mathrm{st}}$ Week & $50.74 \pm 11.35$ & $45.00 \pm 6.55$ & $40.56 \pm 8.40$ & $42.00 \pm 2.54$ & $\begin{array}{c}38.33 \pm 10.7 \\
0\end{array}$ & $40.67+6.78$ \\
\hline $2^{\text {nd }}$ Week & $62.22 \pm 9.64 *$ & $50.67 \pm 8.63$ & $\begin{array}{c}53.90 \pm 8.50 \\
*\end{array}$ & $52.00 \pm 3.90$ & $47.22 \pm 9.74$ & $51.67 \pm 8.40$ \\
\hline $3^{\text {rd }}$ Week & $77.41 \pm 9.94 *$ & $67.00 \pm 5.23$ & $\underset{*}{68.14 \pm 6.81}$ & $67.00 \pm 7.30$ & $58.52 \pm 8.41$ & $63.67 \pm 4.81$ \\
\hline
\end{tabular}

Acupuncture Protocol: Trained Acupuncturist from Xiangya medical hospital, designed the Acupuncture protocol. All cases were given Acupuncture by a wellqualified and experienced acupuncturist.

The Acupuncture methods used in this research were scalp Acupuncture and body Acupuncture (for hemiparalysis ) as follow: The Acupuncture points selected for the paralysis of the upper limb are Jian yu (LI15), zhi gou(sj6)Qu chi (LI 11), Wai guan(S 5), He $\mathrm{gu}(\mathrm{L} 14)$, and/or supplementary points as Bi nao (LI 14), Shou san li (LI 10). The points selected for the paralysis of the lower limb are: $\mathrm{Fu}$ tu(s 32), Xu hai (SP 10), Zu san li (S 36), San yin jiao (SP 6), Tai chong (Liv 3), and/or supplementary points as Feng shi (G20), Tai xi (K3), San yin jiao (SP 6).

Statistical methods for the analysis of data used for motor function and disability function are two sample group $t$ test and Wilcox ion signed rank test. Analyses were performed using SPSS for Windows statistical software (version 13.0).

\section{RESULTS}

Table 2 shows that changes of motor function for RHRH for three groups that acupuncture produces better results than other treatments for stroke and has no affection for RHLH.

Also table 3 shows same improvement of functional disability for RHRH and has no affection for RHLH.

\section{DISCUSSION}

This study demonstrates that a clinical trial of Acupuncture therapy during stroke rehabilitation is 
feasible and succeeds showing additional improvement in motor impairment and disability in RHRH patients and failed to show additional improvement in the RHLH.

The factors have confounded the study and led to a negative result:-

Firstly, duration of the treatment period was shorter in the present trial (three week duration) compared with previous studies.

Secondly, the side of brain lesion affects motor and function recovery post stroke onset. Histological data indicate that there is a greater proportion of neuropil volume in the left hemisphere of primary cortex hand representation area, suggesting the presence of more interconnectivity among neurons ${ }^{[12]}$ Individuals with dominant-side hemi paresis are typically more motivated to use the dominant limb. In contrast, stroke survivors with non-dominant hemiparalysis continue to compensate with the intact, dominant limb. So individuals with dominant limb hemiparalysis have a better therapeutic response to CI therapy than those with non-dominant hemiparalysis, as they appear to be discharged earlier, have better functional and ambulatory abilities and exhibit superior balance control ${ }^{[13]}$

\section{CONCLUSION}

The present study supports both of the previous studies, which indicated that Acupuncture treatment may have a beneficial effect on cerebral infarction as shown in this trial in RHRH and may have no beneficial effect on RHLH in terms of motor function and functional ability.

Also shows clear advantages of Acupuncture treatment of patients in acute phase of cerebral infarction.

\section{REFERENCES}

1. Shi Hong Zhang, Ming Liu, Kjell Asplund and Lin $\mathrm{Li}, 2005$. Acupuncture for Acute Stroke. The American Heart Association. 36:2327-2328

2. Sun Shentian, Li Shurong, Zhu yanzhi et al, 1993. Cliniclstidy on500 cases of cerebro-vascular hemiplegia treated by acupuncture through Bai hui to Qu bin. J of traditional Chinese medicine, 21(4): 305-318.

3. Function recovery in patients with acute cerebral infarction: a randomly controlled trial. J Tradit Chin Med. 21(4): 270-2.
4. Souvik Sen, 2005. Magnetic Resonance Imaging in Acute Stroke [DB/OL]. http://www.emedicine.com/NEURO/topic431.htm.

5. Nudo RJ, Wise BM and SiFuentes F, 1996. Neural substrates for the effects of rehabilitative training on motor recovery after ischemic infarct, J Science, 272:1791-1794.

6. Alexander DN, Cen S and Sullivan KJ, 2004. Effects of Acupuncture Treatment on Poststroke Motor Recovery and Physical Function: A Pilot Study, J Neurorehabil Neural Repair, 18: 259-267.

7. Reinkensmeyer, D.J., Pang, C.T., Nessler, J.A. and Painter, 2002. Web-based telerehabilitation for the upper extremity after stroke, IEEE Trans. on Rehabilitation Engineering, 10(2): 102-108.

8. Chelsea S, Kidwell, Steven Warach, 2003. Acute Ischemic Cerebrovascular Syndrome: Diagnostic Criteria, J Stroke, 34(12): 2995-2998.

9. Scale.Williams LS, Yilmaz EY and Lopez-Yunez $\mathrm{AM}, 2000$. Retrospective assessment of initial stroke severity with the NIH Stroke. American Heart Association, 31(4): 858-62.

10. Patrick D, Lyden MD and Mei Lu, 2001. A Modified National Institutes of Health Stroke Scale for Use in Stroke Clinical Trials Preliminary Reliability and Validity. J Stroke, 32:1310.

11. Nudo RJ, Wise BM, SiFuentes F, Milliken GW. , 1996. Neural substrates for the effects of rehabilitative training on motor recovery after ischemic infarct. Science. 272:1791-1794

12. Kimberley A., 2005. Primary Motor Cortex Asymmetry Is Correlated With Handedness in Capuchin Monkeys (Cebus apella), J American Psychological Association, 119(6): 1701-1704

13. Boris Maslov, Marina Roje-BedekoviÊ and Snjeûana Miökov. , 2002. Acupuncture treatment in facial palsy clinical observations, J Acta Clin Croat, 43:275-279. 